\title{
ADOTE UM SORRISO: O RESGATE DA AUTOESTIMA DE IDOSOS INSTITUCIONALIZADOS
}

\section{ADOPT A SMILE: THE RESCUE OF THE SELF-ESTIMATE OF INSTITUTIONALIZED ELDERLY}

\author{
Danilo Vieira Barbosa* \\ ORCID: https://orcid.org/0000-0003-0386-8895 \\ Waleska Fernanda Souto Nóbrega** \\ ORCID: https://orcid.org/0000-0001-8140-4063 \\ Gustavo Correia Basto da Silva*** \\ ORCID: https://orcid.org/0000-0002-6081-2540 \\ Osires de Medeiros Melo Neto ${ }^{* * * *}$ \\ ORCID: https://orcid.org/0000-0002-2535-0969 \\ Luciana Ellen Dantas Costa**** \\ ORCID: https://orcid.org/0000-0003-4476-7900 \\ Faldryene de Souza Queiroz Feitosa ${ }^{* * * * *}$ \\ ORCID: https://orcid.org/0000-0001-8531-871X
}

\section{RESUMO:}

Objetiva-se, com este estudo, descrever as experiências adquiridas por acadêmicos durante a vigência do projeto de extensão "Adote um Sorriso", a partir do convívio com idosos de instituições de longa permanência. Atuaram no projeto 12 graduandos do curso de Odontologia da Universidade Federal de Campina Grande, sob a supervisão de uma docente, no período de abril a dezembro de 2017. Pôde-se observar, na maioria dos idosos institucionalizados, um expressivo déficit de autoestima, aspectos relacionados à perda de identidade e uma necessidade, muitas vezes exacerbada, de serem notados e ouvidos com a atenção devida. Após o término do período de convivência, foi possível concluir que o projeto extensionista alcançou seus principais objetivos, por ter proporcionado o estabelecimento de vínculos entre os idosos institucionalizados com os acadêmicos de Odontologia, além do resgate da autoestima dos idosos, e despertar um lado mais humanizado no olhar dos alunos participantes.

Palavras chave: Assistência a idosos; Qualidade de vida; Relações comunidade-instituição; Odontologia comunitária.

\begin{abstract}
:
The objective of this study is to describe the experiences acquired by undergraduate students during the development of the university outreach project "Adopt a Smile", from the interaction with elderly people residing in long-term institutions. Twelve undergraduate students from the Dentistry course at the Federal University of Campina Grande worked under the supervision of a professor from April to December 2017. Most institutionalized elderly people showed a significant deficit in self-esteem, aspects related to loss of identity and a need, often exacerbated, to be noticed and listened to with due attention. After the end of the coexistence period, it was possible to conclude that the outreach project achieved its main objectives, as it provided the establishment of bonds between the institutionalized elderly people and the dentistry students in that group. It also rescued the elderly's self-esteem, and awakened a more humanized side in the eyes of the participating students.
\end{abstract}

Keywords: Assistance to the elderly; Quality of life; Community-institution relations; Community dentistry.

\footnotetext{
${ }^{*}$ Graduação. Universidade Federal de Campina Grande (UFPB), Patos - PB, Brasil. E-mail: danilo_vieira23@hotmail.com

** Aluna de Doutorado da Universidade Estadual da Paraíba (UEPB), Campina Grande - PB, Brasil. E-mail: drawaleskasouto@gmail.com

*** Aluno de Doutorado da Universidade Estadual da Paraíba (UEPB), Campina Grande - PB, Brasil. E-mail: gugacorreiaa@gmail.com

**** Aluno de Mestrado da Universidade Federal de Campina Grande (UFCG), Campina Grande - PB, Brasil. E-mail: osiresdemedeiros@gmail.com

${ }^{* * * * *}$ Professora da Universidade Federal de Campina Grande (UFCG), Patos - PB, Brasil. E-mail: ellendantascosta@yahoo.com.br

****** Professora da Universidade Federal de Campina Grande (UFCG), Patos - PB, Brasil. E-mail: falqueiroz@hotmail.com
} 


\section{Introdução}

O processo de envelhecimento, causado por alterações moleculares e celulares, é progressivo e acarreta diversas alterações e desgastes tanto da capacidade física, psicossocial, quanto de autoestima, que podem comprometer a qualidade de vida dos idosos (MOURÃO et al., 2010; ASSIS et al., 2016). No Brasil, o número de idosos vem crescendo progressivamente, gerando o aumento da demanda por instituições de longa permanência para eles. Porém, nesses ambientes, as práticas educativas em saúde e práticas recreativas são discretas ou, até mesmo, inexistentes (GAIÃO et al., 2005).

$\mathrm{O}$ crescimento da população idosa faz com que seja essencial que projetos e ações lúdicas e recreativas sejam desenvolvidos nas instituições que os abrigam para que se possa garantir a melhoria dos problemas consequentes do envelhecimento (ASSIS et al., 2016; LAGO et al., 2017).

Em um estudo, objetivando analisar a autopercepção de saúde de idosos institucionalizados, observou-se que a situação em que se encontram, ocasionam sentimentos de saudade e exclusão que interferem diretamente no conceito de saúde desses indivíduos (FREIRE et al., 2005).

A saúde bucal de idosos institucionalizados também é um aspecto comprometido, quando se compara a idosos não institucionalizados. O índice CPO-D é geralmente alto, com elevado índice de dentes perdidos e cariados, assim como alta necessidade de prótese (SOUZA et al., 2010). A saúde bucal deficiente interfere negativamente na qualidade de vida desses indivíduos (CORNEJO et al., 2013).

Estudos demonstram que, para atender de forma adequada às necessidades da população idosa, é imprescindível que haja uma integração dos cursos de graduação ao conteúdo de odontogeriatria. Além disso, o envolvimento dos alunos em atividades práticas de educação em saúde, atividades lúdicas, dentre outras, beneficia demasiadamente a população idosa em diversos aspectos, tais como lazer, autocuidado e melhoria da autoestima, beneficiando os cuidadores, profissionais e alunos envolvidos em conhecimento teórico e prático sobre o processo de envelhecimento (MOIMAS et al., 2011; ARVELOS et al., 2011).

Idosos institucionalizados têm necessidade de estímulos recreativos constantes para que haja a manutenção de sua qualidade de vida e para que as suas funções cognitivas não declinem em virtude da institucionalização (LEITE et al., 2009). Nesse aspecto, algumas instituições que oferecem atividades recreativas têm alcançado sucesso na estimulação da coordenação motora, criatividade e raciocínio dos idosos residentes (LIMA et al., 2010). Neste sentido, algumas atividades recomendadas quando se pretende promover lazer ao idoso institucionalizado são: práticas religiosas, relações interpessoais, ginástica, práticas manuais e artesanais (ALVES et al., 2013).

A extensão universitária surgiu para preencher a lacuna deixada pelo ensino e pela pesquisa no sentido de transformar a realidade dos jovens brasileiros no atual cenário de exclusão social do país (FREIRE, 2011). Busca-se utilizar a extensão como forma de abrir as portas da universidade para a comunidade e, através de processos educativos, estabelecer vínculo entre estes (NUNES et al., 2011).

É de fundamental importância, nos tempos atuais, mediar a formação em saúde através da ferramenta de extensão universitária, a qual articula a universidade, os gestores e a comunidade, a fim de proporcionar repercussões positivas no âmbito pessoal e profissional dos jovens (BISCARDE et al., 2014). Neste sentido, o presente trabalho tem como objetivo relatar a experiência de alunos do curso de Odontologia da Universidade Federal de Campina Grande nas ações do projeto de extensão "Adote um Sorriso: um resgate da autoestima de idosos institucionalizados da cidade de Patos-PB", cujo foco principal era resgatar a autoestima de idosos institucionalizados, em longa permanência, da cidade de Patos - PB. 


\section{Material e métodos}

Trata-se de um estudo descritivo, com abordagem qualitativa, do tipo relato de experiência. A busca bibliográfica foi realizada nas bases de dados Scielo e BVS, nas línguas portuguesa e inglesa. O projeto foi aprovado pela Pró-Reitoria de Pesquisa e Extensão e teve vigência durante todo o ano de 2017.

O "Adote um Sorriso" abrangeu o número total de 50 idosos pertencentes a duas instituições de longa permanência da cidade de Patos - PB: o Lar dos Idosos Jesus de Nazaré e o Lar dos Velhinhos do Jatobá.

Atuaram efetivamente no projeto 12 graduandos do curso de Odontologia da UFCG sob a supervisão de uma docente do quadro efetivo da instituição. Eles foram responsáveis pela realização de visitas semanais às instituições entre os meses de abril e dezembro de 2017. Cada visita tinha, em média, 2 horas de duração, de forma a não interferir na rotina dos abrigos, e a cada semana era alternado o turno em que se realizaria a visita. Como forma de estabelecer um diagnóstico situacional acerca do perfil dos idosos, foi aplicado um formulário, contendo dados socioeconômicos (idade, gênero, escolaridade, estado civil), tempo de institucionalização, número de filhos, se tem contato com a família, se recebe visitas, hábito de etilismo e tabagismo, portador de doenças, deambulação e o Índice de Katz (KATZ et al., 1963).

Como método quantitativo de avaliação, ao longo do projeto foi aplicado um formulário, contendo informações sobre a opinião dos idosos em relação à convivência interna, sobre as atividades preexistentes voltadas para o lazer e, finalmente, sobre a sua opinião em relação à participação dos alunos de Odontologia nas atividades desenvolvidas na instituição.

A pesquisa seguiu os preceitos éticos estabelecidos pela Resolução 466/2012, sendo submetida e aprovada pelo Comitê de Ética e Pesquisa por meio do Parecer n. 3.323.669.

\section{Relato de experiência}

Inicialmente, as visitas tinham o objetivo de proporcionar aos graduandos a oportunidade de conhecer a realidade de cada instituição, levando em consideração suas normas e rotinas, buscando entender como se dava a interação entre idosos e o modo como tal influenciava dentro daquele cotidiano, mas principalmente primou-se pela construção de um vínculo com os idosos institucionalizados, bem como com funcionários das instituições, pois isso seria primordial para o bom andamento do projeto.

Pôde-se observar, na maioria dos idosos institucionalizados, um expressivo déficit de autoestima, aspectos de perda de identidade e uma necessidade, muitas vezes exacerbada, de serem notados e ouvidos com a atenção devida. Diante desta perspectiva, prontamente iniciou-se o processo de estabelecimento de vínculo, através de conversas que proporcionassem, de fato, o melhor entendimento de suas necessidades de interação.

Foi estabelecida a realização de atividades de cunho social, com o intuito de assistir os idosos institucionalizados, e, assim, atender suas necessidades e anseios sociais e emocionais, individual e coletivamente, tendo em vista que se buscava a recuperação de uma identidade individual, bem como a interação coletiva entre eles próprios e os acadêmicos. Além disso, realizaram-se atividades voltadas para a promoção e prevenção da saúde geral e bucal.

Como forma de se estabelecer uma melhor descrição e entendimento de sua dinâmica, as atividades do projeto foram agrupadas em 9 categorias:

- Escuta de relatos, experiências, queixas e desenvolvimento de conversas espontâneas com os idosos;

- Estimulação da memória, integração, aumento da autoestima e valorização dos idosos;

- Organização de eventos culturais e festejos em datas comemorativas; 
- Atividades estéticas e "momento da beleza";

- Ações de promoção e prevenção da saúde geral e bucal;

- Realização de exames preventivos na cavidade oral e tecidos moles da face;

- Encaminhamento dos idosos com lesões suspeitas;

- Estabelecimento de diagnóstico situacional;

- Avaliação das atividades desenvolvidas.

O desenvolvimento de conversas espontâneas, e através dessas, ouvir os relatos e experiências dos idosos, permitiu um contato mais próximo entre eles e os acadêmicos. A valorização deles, assim como o estímulo de sua memória e aumento de sua autoestima foram alcançados com o auxílio de artifícios como jogos, músicas, religiosidade, cultura popular e, acima de tudo, uma alta gama de conversas e atenção individual. Esse conjunto de atividades propiciou o estabelecimento de um vínculo sólido com cada um dos idosos, o que culminou na oportunidade de seguir para o estágio das ações coletivas, onde a interação foi indescritível.

Nas ações coletivas, sempre buscou- se a devolução da identidade individual. Um dos artifícios utilizados para isso foi o estabelecido como padrão "momento da beleza", onde os homens tiveram barba feita (Figura 1) e oportunamente os cabelos cortados, ao passo que as mulheres tiveram unhas feitas e pintadas, foram maquiadas e oportunamente tiveram os cabelos produzidos. Paralelamente ao momento da beleza, sempre houve ações voltadas para a diversão e interação, com auxílio de dinâmicas com músicas e jogos que promovessem o reforço da memória e a possibilidade de se manter um diálogo atencioso, reforçando-se, assim, o vínculo idoso-acadêmico, proporcionando-lhes o sentimento de apoio constante.

Foram realizadas atividades em consonância com cada época do ano e suas comemorações habituais, como: dia das mães, festejos juninos (Figura 2), dia dos pais e Natal (Figura 3). Em tais eventos, buscou-se o resgate da memória afetiva, da alegria que tais momentos são popularmente conhecidos por proporcionarem. Músicas regionais, quadrilhas e troca de presentes são exemplos de abordagens realizadas durante as atividades supracitadas.

Figura 1 - Realização de cuidados em higiene bucal.

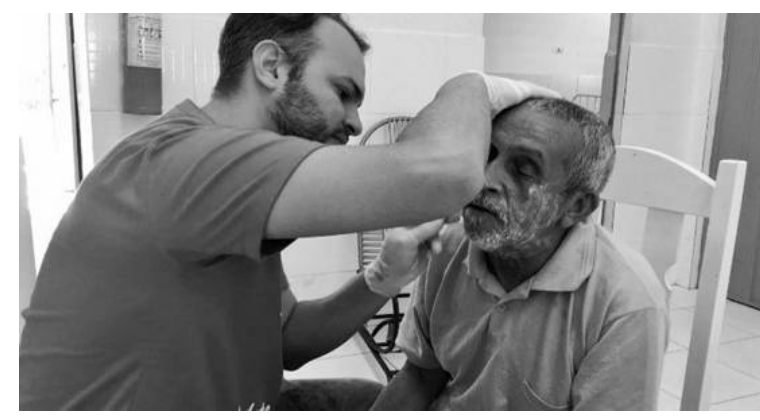

Figura 2 - Confraternização junina.

Figura 3 - Atividades lúdicas em alusão ao período natalino.
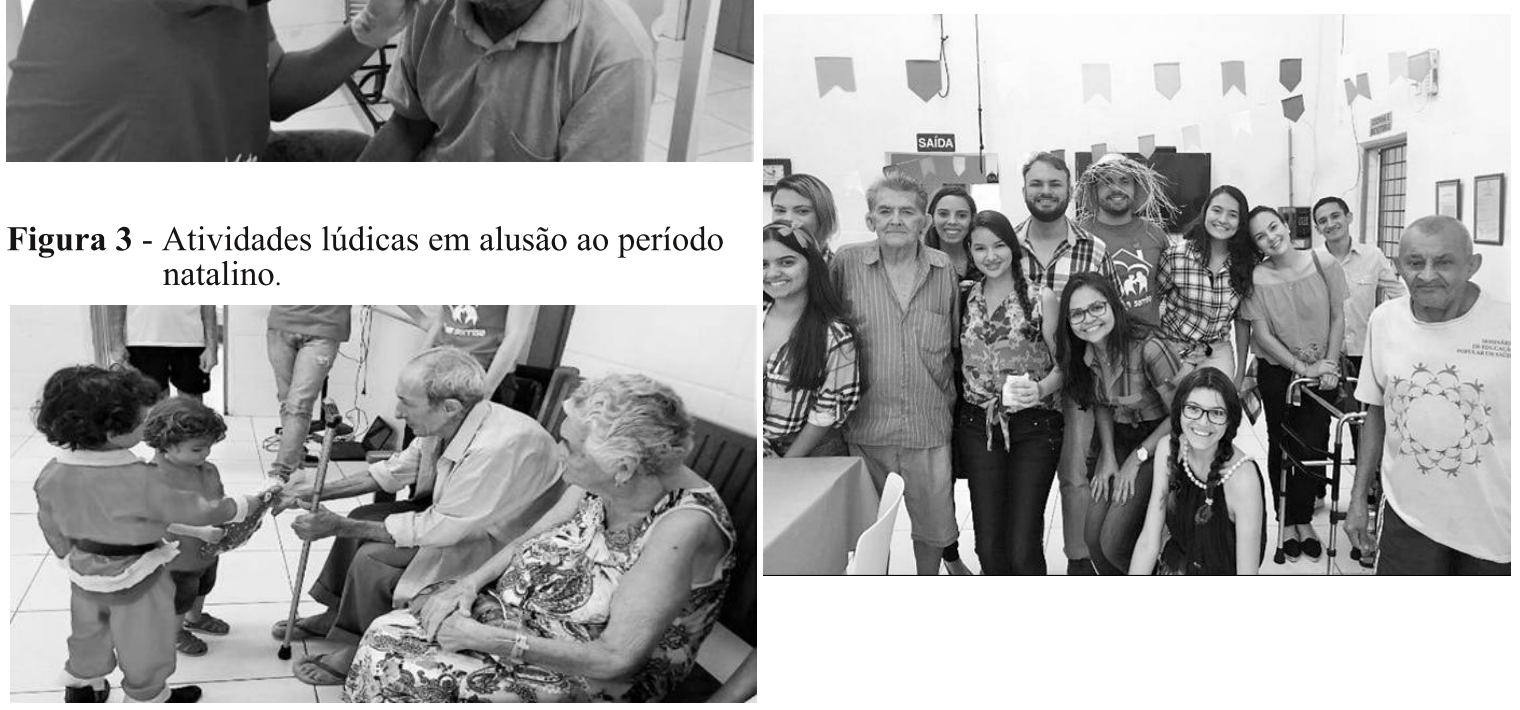

Fonte: Autores. 
Com a evolução das atividades, realizaram-se ações de promoção e prevenção da saúde geral e bucal. Por meio de atividades lúdicas, teatralização, conversas e oficinas, abordaram-se vários assuntos de saúde, contribuindo para o processo de empoderamento dos idosos, o que resultou, dentro das possibilidades de cada um, em autocuidado. Além disso, foram acrescentadas informações aos conhecimentos dos cuidadores, sendo possível formar agentes multiplicadores dessas práticas de promoção e prevenção de saúde.

Os extensionistas realizaram exames clínicos na cavidade oral e tecidos moles das faces de cada idoso, com o intuito de detectar lesões cancerígenas e pré-cancerígenas. No caso de detecção, foi realizado o encaminhamento do idoso acometido para o setor de Estomatologia da Clínica-escola de Odontologia da Universidade Federal de Campina Grande - UFCG.

Houve, ainda, a realização de um diagnóstico situacional dos idosos, por meio de consulta aos seus prontuários e entrevista direta com eles e seus cuidadores, dependendo da capacidade de resposta de cada idoso. Esse diagnóstico englobou os dados socioeconômicos, tempo de institucionalização, número de fillhos, se tem contato com a família, se recebe visitas, hábito de etilismo e tabagismo, portador de doenças, deambulação e o Índice de Katz (1963). Esse índice se baseia numa avaliação da independência ou dependência funcional dos pacientes para banhar-se, vestir-se, usar o sanitário, mobilizar-se, ser continente e comer sem ajuda.

Verificou-se, nessa etapa, que a maioria dos idosos (32\%) era de solteiros, que estavam nas instituições em média de 1 a 5 anos (62\%), não eram alfabetizados (44\%), trabalharam durante a vida (72\%), tiveram filhos (56\%), recebiam visitas da família (60\%), não tinham o hábito de fumar (62\%), e não bebiam (70\%).

Quanto ao estado de saúde e presença de morbidades, 28\% tinham hipertensão, $22 \%$ relataram ter diabetes, $6 \%$ apresentavam sequelas de Acidentes Vascular Cerebral (AVC), $4 \%$ possuíam Alzheimer e $8 \%$ eram acamados. Durante a entrevista, 20\% dos idosos relataram ser tristes e $4 \%$ muito tristes.

De acordo com o Índice Katz (KATZ et al., 1963), 44\% dos idosos não necessitavam de assistência no banho, 44\% precisavam de alguma assistência ao se vestir, $36 \%$ precisavam de assistência ao usar o banheiro, além de $22 \%$ terem acidentes ocasionais de incontinência urinária ou fecal, e 10\% eram impossibilitados de levantar-se, $30 \%$ necessitavam de assistência para tal, e 6\% necessitavam de assistência total para se alimentarem.

No decorrer do tempo, em meio à realização das atividades e com a concretização do vínculo, pôde-se perceber a aparição de uma face surpreendentemente feliz em cada idoso, o resgate da capacidade de sorrir e a demonstração, tudo reforçado por relatos verbais e uma elevação de autoestima, além da clara satisfação em sentirem-se mais uma vez notados e importantes por alguém que eles estimam.

Os acadêmicos passaram a demonstrar uma evolução extremamente satisfatória no que se refere à humanização em saúde, ao estabelecer, de fato, laços afetivos com os idosos, com relações, ações e comportamentos que muitas vezes remeteram a uma substituição de alguma figura da própria família, inclusive com fortes e positivas reações emocionais.

Ao fim da vigência do projeto, a equipe se reuniu para refletir sobre todas as melhorias e evoluções pessoais supracitadas (Figura 4). Estas proporcionaram a oportunidade de auxiliar também os funcionários das instituições, que, por diversas vezes, são sobrecarregados diante da expectativa de suprir as próprias metas institucionais e humanas, a alcançar os resultados mais satisfatórios e holísticos. Ficou nítida a gratidão e valorização pelas ações realizadas em ambas as instituições de longa permanência. 
Figura 4 - Encerramento das atividades.

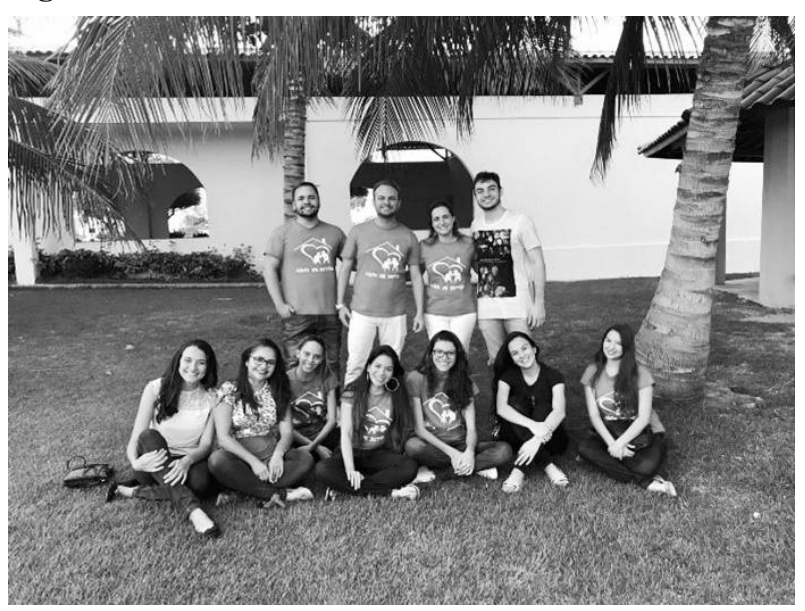

Fonte: Autores.

\section{Conclusão}

Após todo o exposto, é possível concluir que o projeto de extensão "Adote um Sorriso" alcançou seus objetivos, pois proporcionou o estabelecimento de um vínculo dos idosos e instituições com os acadêmicos de Odontologia, resgatou a autoestima e identidade dos idosos e despertou um lado mais humanizado no olhar dos acadêmicos participantes. Conseguiu, ainda, ampliar o olhar profissional de cada participante para questões mais simples, como a importância do ouvir, do tocar, de promover a sensação de ser importante, que nem sempre são consideradas.

Como limitação, cabe destacar a quantidade e duração das visitas, que devido ao elevado número de necessidades individuais, tornavam- se muito curtas, mas que foram importantes para a comprovação da validade acadêmica, social, profissional e principalmente humana do projeto.

Depois de uma experiência tão produtiva e enriquecedora, seria quase impossível que o projeto não continuasse, seguindo com a perspectiva de manutenção da qualidade a ampliação de ações, visitas e número de participantes.

\section{Referências}

ALVES DE MOURA, G. de Souza, L. K. Práticas de lazer de idosos institucionalizados. Movimento, v. 19, n. 4, p. 69-93, 2013.

ARVELOS, E.S.; LANDIM, L.M.; SILVA, M.R.; MARGNABOSCO, P.; DOS ANJOS, A.C.Y. Projeto "Faça um idoso feliz": Promoção de atividades de lazer a idosos institucionalizados. Em Extensão, Uberlândia, v. 10, n. 1, p. 139-145, 2011.

ASSIS, B.V.; FARIA, L.P.; DE ASSIS, R.M. et al. Atividades Recreativas para idosos institucionalizados: projeto de extensão do curso de Educação Física/UFG/REJ. In: SEMANA DE LICENCIATURA, 13., 2016, Jataí. Anais... Jataí, GO, 2016. 
BISCARDE, D.G.S.; PEREIRA-SANTOS, M.; BITTENCOURT, L. Formação em saúde, extensão universitária e Sistema Único de Saúde (SUS): conexões necessárias entre conhecimento e intervenção centradas na realidade e repercussões no processo formativo. Espaço Aberto, Interface, v. 18, n.48, p.177-186, 2014.

CORNEJO, M.; PEREZ, G.; DE LIMA, K.C. et al. Oral Health-Related Quality of Life in institutionalized elderly in Barcelona (Spain). Med Oral Patol Oral Cir Bucal., v. 18, n. 2, p. 285-92, 2013.

FREIRE JR., R.C.; TAVARES, M.F.L. Health from the viewpoint of institutionalized senior citizens: getting to know and value their opinion. Interface - Comunic., Saúde, Educ., v.9, n.16, p.147-58, 2005.

FREIRE, S.M. Desafios da extensão universitária na contemporaneidade. Revista Conexão UEPG. v. 7, n. 1, p. 8-15, 2011.

GAIÃO, L.R.; ALMEIDA, M.E.L.; HEUKELBACH, J. Perfil epidemiológico da cárie dentária, doença periodontal, uso e necessidade de prótese em idosos residentes em uma instituição na cidade de Fortaleza, Ceará. Rev Bras Epidemiol., v. 8, n. 3, p. 316-23, 2005.

KATZ, S.; FORD, A.B.; MOSKOWITZ, R.W.; JACKSON, B.A.; JAFFE, M.W. Studies of illness in the aged. The index of ADL: a standardized measure of biological and psychosocial function. JAMA, v. 185, n. 3, p. 914-9, 1963.

LAGO, J.D.; FAIS, L.M.G. et al. Educational program in oral health for caregivers on the oral hygiene of dependent elders. Rev Odontol UNESP, v. 46, n. 5, p. 284-291, 2017.

LEITE, B.F.L.; SALVADOR, D.H.Y.; ARAÚJO, C.L.O. Avaliação cognitiva dos idosos institucionalizados. Kairós, v.12, n. 1, p. 247-256, 2009.

LIMA, D.L. et al. Envelhecimento e qualidade de vida de idosos institucionalizados. RBCEH, Passo Fundo, v. 7, n. 3, p. 346-356, 2010

MOIMAS, S.A.S.; GARBIN, C.A.S.; SILVA, M.M. et al. Projeto "Sempre Sorrindo": 10 anos de atenção ao idoso institucionalizado. Revista Brasileira de Pesquisa em Saúde, v. 13, n. 1, p. 46-51, 2011.

MOURÃO. C.A.; SILVA, N.M. Influência de um programa de atividades físicas recreativas na autoestima de idosos institucionalizados. RBCEH, Passo Fundo, v. 7, n.3, p. 324-334, 2010.

NUNES, A.L.P.F.; SILVA, M.B.C. A extensão universitária no ensino superior e a sociedade. Mal-Estar e Sociedade. v. 4, n. 7, p. 119-133, 2011.

SOUZA, E.H.A. et al. Impacto da saúde bucal no cotidiano de idosos institucionalizados e não institucionalizados da cidade do Recife (PE, Brasil). Ciência\&SaúdeColetiva, v. 15, n. 6, p.2955-2964, 2010. 